\title{
A Comparative Study of Divergence-Cleaning Techniques for Multi-Dimensional MHD Schemes*)
}

\author{
Takahiro MIYOSHI and Kanya KUSANO ${ }^{1)}$ \\ Hiroshima University, Higashi-Hiroshima 739-8526, Japan \\ ${ }^{1)}$ Nagoya University, Nagoya 464-8601, Japan
}

(Received 22 December 2010 / Accepted 13 May 2011)

\begin{abstract}
Several divergence-cleaning techniques for multi-dimensional Godunov-type magnetohydrodynamic schemes are comparatively investigated. We also propose a new divergence-cleaning technique that is improved from an earlier projection method to improve the robustness.
\end{abstract}

(c) 2011 The Japan Society of Plasma Science and Nuclear Fusion Research

Keywords: MHD, Godunov-type scheme, divergence-cleaning method, divergence-free method

DOI: $10.1585 /$ pfr.6.2401124

\section{Introduction}

Godunov-type schemes for magnetohydrodynamics (MHD) have been developed for decades [1,2] and recently have been widely applied to space and astrophysical problems [3,4]. Although Godunov-type MHD schemes can precisely capture shocks and discontinuities without numerical oscillations in one dimension, multidimensional extensions are not straightforward because the divergence-free condition of the magnetic field is difficult to maintain in multi-dimensional MHD schemes. Because of the numerical divergence of the magnetic field, multi-dimensional simulation runs are sometimes unusually terminated. Therefore, the development of divergencecleaning numerical techniques remains an important challenge in computational MHD. Although excellent comparative studies of divergence-cleaning and/or essentially divergence-free techniques have been carried out [5,6], a number of important ideas [7, 8] have not been considered in those studies. In this paper, divergence-cleaning and divergence-free techniques are comparatively studied with emphasis on the robustness of the methodology. We adopt the second-order total variation diminishing (TVD) Runge-Kutta method for time integration [9] and the finite volume method using the second-order Harten-Laxvan Leer-Discontinuities (HLLD) approximate Riemann solver for space discretization [2].

\section{Divergence-Cleaning Methods}

The following divergence-cleaning and divergencefree techniques are investigated. For simplicity, a uniform Cartesian grid is adopted, as shown in Fig. 1.

Projection method: In the projection method [10], the magnetic field $\boldsymbol{B}^{*}$ computed by the multi-dimensional

author'se-mail: miyoshi@sci.hiroshima-u.ac.jp

*) This article is based on the presentation at the 20th International Toki Conference (ITC20).

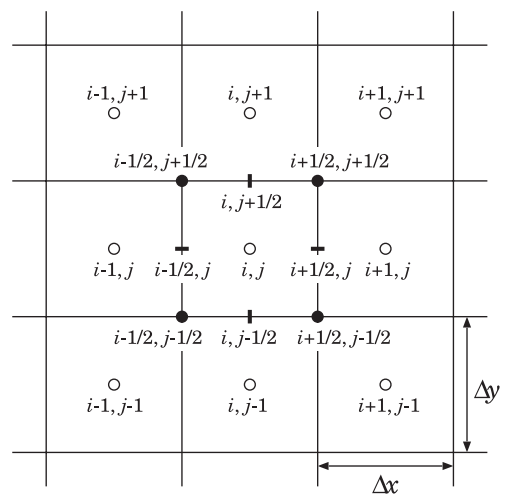

Fig. 1 Grid structure. White circles (०), black circles $(\bullet)$, and thick lines (-) indicate cell centers, cell edges, and cell faces, respectively.

MHD scheme is projected into the subspace of divergencefree fields after every time step as

$$
\boldsymbol{B}=\boldsymbol{B}^{*}+\nabla \phi
$$

where $\phi$ must satisfy a Poisson equation as

$$
\nabla^{2} \phi=-\nabla \cdot \boldsymbol{B}^{*}
$$

Eq. (1) is directly discretized as

$$
\begin{aligned}
& B_{x, i, j}=B_{x, i, j}^{*}+\frac{\phi_{i+1, j}-\phi_{i-1, j}}{2 \Delta x}, \\
& B_{y, i, j}=B_{y, i, j}^{*}+\frac{\phi_{i, j+1}-\phi_{i, j-1}}{2 \Delta y} .
\end{aligned}
$$

If $\phi_{i, j}$ satisfies the condition

$$
\begin{array}{r}
\frac{\phi_{i+2, j}-2 \phi_{i, j}-\phi_{i-2, j}}{4 \Delta x^{2}}+\frac{\phi_{i, j+2}-2 \phi_{i, j}-\phi_{i, j-2}}{4 \Delta y^{2}} \\
=-\frac{B_{x, i+1, j}^{*}-B_{x, i-1, j}^{*}}{2 \Delta x}-\frac{B_{y, i, j+1}^{*}-B_{y, i, j-1}^{*}}{2 \Delta y},
\end{array}
$$

the discrete divergence-free condition such that $\left(B_{x, i+1, j}-\right.$ $\left.B_{x, i-1, j}\right) / 2 \Delta x+\left(B_{y, i, j+1}-B_{y, i, j-1}\right) / 2 \Delta y=0$ is strictly assured. 
Face-projection method: As discussed in Ref. [6], Eq. (5) sometimes leads to a checkerboard instability or an odd-even decoupling. Although Eq. (2) is discretized using neighbor grids instead of Eq. (5), the discrete divergencefree condition is not satisfied. We propose herein another variant of the projection method. The original projection method implicitly assumes that the magnetic field at a cell face is given by the arithmetic mean at adjacent cells, e.g., $\boldsymbol{b}_{i+1 / 2, j}=\left(\boldsymbol{B}_{i+1, j}+\boldsymbol{B}_{i, j}\right) / 2$. On the other hand, the present method assumes that

$$
b_{x, i+1 / 2, j}=\frac{B_{x, i+1, j}+B_{x, i, j}}{2}+\frac{\phi_{i+1, j}-\phi_{i, j}}{\Delta x},
$$

and

$$
b_{y, i, j+1 / 2}=\frac{B_{y, i, j+1}+B_{y, i, j}}{2}+\frac{\phi_{i, j+1}-\phi_{i, j}}{\Delta y} .
$$

Using the discrete divergence-free condition such that $\left(b_{x, i+1 / 2, j}-b_{x, i-1 / 2, j}\right) / \Delta x+\left(b_{y, i, j+1 / 2}-b_{y, i, j-1 / 2}\right) / \Delta y=0$,

$$
\begin{array}{r}
\frac{\phi_{i+1, j}-2 \phi_{i, j}-\phi_{i-1, j}}{\Delta x^{2}}+\frac{\phi_{i, j+1}-2 \phi_{i, j}-\phi_{i, j-1}}{\Delta y^{2}} \\
=-\frac{B_{x, i+1, j}-B_{x, i-1, j}}{2 \Delta x}-\frac{B_{y, i, j+1}-B_{y, i, j-1}}{2 \Delta y} .
\end{array}
$$

In this method, before every time step, the normal component of the magnetic field at each cell face is calculated by Eqs. (6), (7), and (8). Then, the numerical flux at the cell face is computed using the divergence-free magnetic field, where the tangential fields at both sides of the face are reconstructed by some interpolation technique such as the MUSCL method. Because the magnetic field at the cell center evolves with time by the Godunov-type scheme without correction, the energy conservation is consistent. On the other hand, the original projection method changes the magnetic energy at the projection step.

Flux-CT method: The constrained transport (CT) method is constructed on a staggered grid within which Stokes' theorem is applied to the induction equation on each cell face. Thus, the increments of the magnetic field at cell faces are given by

$$
\begin{aligned}
& \Delta b_{x, i+1 / 2, j}=-\frac{\Delta t}{\Delta y}\left(E_{z, i+1 / 2, j+1 / 2}-E_{z, i+1 / 2, j-1 / 2}\right), \\
& \Delta b_{y, i, j+1 / 2}=\frac{\Delta t}{\Delta x}\left(E_{z, i+1 / 2, j+1 / 2}-E_{z, i-1 / 2, j+1 / 2}\right)
\end{aligned}
$$

in two dimensions. Because the numerical fluxes for the induction equation are regarded as the electromotive force (EMF) at cell faces, e.g.,

$$
E_{z, i+1 / 2, j}=-F_{x, i+1 / 2, j}^{B_{y}}, E_{z, i, j+1 / 2}=F_{y, i+1 / 2, j}^{B_{x}},
$$

where $F_{x}^{B_{y}}$ and $F_{y}^{B_{x}}$ are the $x$-component of the flux for $B_{y}$ and the $y$-component for $B_{x}$, respectively, in the induction equation, the EMF at a cell edge may be evaluated by an average of the numerical fluxes as follows [11]:

$$
\begin{aligned}
E_{z, i+1 / 2, j+1 / 2}=\frac{1}{4}( & E_{z, i+1 / 2, j}+E_{z, i+1 / 2, j+1} \\
& \left.+E_{z, i, j+1 / 2}+E_{z, i+1, j+1 / 2}\right) .
\end{aligned}
$$

The cell center value of the magnetic field can be given by the arithmetic mean at adjacent cell faces, for example.

HLL-flux-CT method: As pointed out in Ref. [8], the flux-CT method is not reduced to the one-dimensional scheme for plane-parallel grid-aligned flows. Instead of Eq. (12), another variant of the EMF can be estimated as

$$
\begin{aligned}
& E_{z, i+1 / 2, j+1 / 2} \\
& =\frac{1}{4}\left(E_{z, i+1 / 2, j}+E_{z, i+1 / 2, j+1}+E_{z, i, j+1 / 2}+E_{z, i+1, j+1 / 2}\right) \\
& +\frac{\Delta y}{8}\left[\left(\frac{\partial E_{z}}{\partial y}\right)_{i+1 / 2, j+1 / 4}-\left(\frac{\partial E_{z}}{\partial y}\right)_{i+1 / 2, j+3 / 4}\right] \\
& +\frac{\Delta x}{8}\left[\left(\frac{\partial E_{z}}{\partial x}\right)_{i+1 / 4, j+1 / 2}-\left(\frac{\partial E_{z}}{\partial x}\right)_{i+3 / 4, j+1 / 2}\right]
\end{aligned}
$$

The derivatives of the EMF on the cell face may be given by numerical fluxes of the evolution equations for the derivatives of the magnetic field [8]. Moreover, the derivatives of the EMF are replaced by the derivatives in the upwind side [8]. Here we apply the HLL fluxes, e.g., as

$$
\begin{aligned}
& \left(\frac{\partial E_{z}}{\partial y}\right)_{i+1 / 2, j+1 / 4} \\
& =\frac{2}{\Delta y} \frac{s_{\mathrm{r}, i+1 / 2, j}\left(E_{z, i, j+1 / 2}-E_{z, i, j}\right)}{s_{\mathrm{r}, i+1 / 2, j}-s_{1, i+1 / 2, j}} \cdots \\
& \ldots \frac{-s_{1, i+1 / 2, j}\left(E_{z, i+1, j+1 / 2}-E_{z, i+1, j}\right)}{\ldots} \\
& \ldots \frac{+s_{\mathrm{r}, i+1 / 2, j} S_{1, i+1 / 2, j}\left(-B_{y, i+1, j+1 / 2}+B_{y, i+1, j}\right.}{\ldots} \\
& \ldots \frac{\left.+B_{y, i, j+1 / 2}-B_{y, i, j}\right)}{} \\
& \left(\frac{\partial E_{z}}{\partial x}\right)_{i+1 / 4, j+1 / 2} \\
& =\frac{2}{\Delta x} \frac{s_{\mathrm{r}, i, j+1 / 2}\left(E_{z, i+1 / 2, j}-E_{z, i, j}\right)}{s_{\mathrm{r}, i, j+1 / 2}-s_{1, i, j+1 / 2}} \ldots \\
& \ldots \frac{-s_{1, i, j+1 / 2}\left(E_{z, i+1 / 2, j+1}-E_{z, i, j+1}\right)}{\ldots} . \\
& \ldots \frac{+s_{\mathrm{r}, i, j+1 / 2} S_{1, i, j+1 / 2}\left(B_{x, i+1 / 2, j+1}-B_{x, i, j+1}\right.}{\cdots} \\
& \ldots \frac{\left.-B_{x, i+1 / 2, j}+B_{x, i, j}\right)}{}
\end{aligned}
$$

where $s_{\mathrm{r}}$ and $s_{1}$ are the maximum and minimum wave speeds at the cell face [2]. This algorithm adopts an HLLweighted average of the derivatives of the EMF and reproduces the original one-dimensional scheme for planeparallel, grid-aligned flows.

Eight-wave method: The eight-wave method requires a source term in proportion to the divergence of the magnetic field [12]. For example, the induction equation with a source term as

$$
\frac{\partial \boldsymbol{B}}{\partial t}+\nabla \cdot(\boldsymbol{V} \boldsymbol{B}-\boldsymbol{B} \boldsymbol{V})=-\boldsymbol{V}(\nabla \cdot \boldsymbol{B})
$$


is explicitly solved. The divergence of Eq. (16) becomes

$$
\frac{\partial(\nabla \cdot \boldsymbol{B})}{\partial t}+\nabla \cdot[\boldsymbol{V}(\nabla \cdot \boldsymbol{B})]=0 .
$$

This means that the divergence of the magnetic field is advected with a local speed.

Hyperbolic divergence-cleaning method: In this method, an additional scalar evolution equation is solved and coupled with the induction equation to become totally hyperbolic as follows [7].

$$
\begin{aligned}
& \frac{\partial \boldsymbol{B}}{\partial t}+\nabla \cdot(\boldsymbol{V} \boldsymbol{B}-\boldsymbol{B} \boldsymbol{V}+\psi \boldsymbol{I})=0, \\
& \frac{\partial \psi}{\partial t}+c_{\mathrm{h}}^{2} \nabla \cdot \boldsymbol{B}=-\frac{c_{\mathrm{h}}^{2}}{c_{\mathrm{p}}^{2}} \psi,
\end{aligned}
$$

where $c_{\mathrm{h}}$ and $c_{\mathrm{p}}$ indicate uniformly constant free parameters. From these equations, a telegraph equation,

$$
\frac{\partial^{2}(\nabla \cdot \boldsymbol{B})}{\partial t^{2}}+\frac{c_{\mathrm{h}}^{2}}{c_{\mathrm{p}}^{2}} \frac{\partial(\nabla \cdot \boldsymbol{B})}{\partial t}-c_{\mathrm{h}}^{2} \nabla^{2}(\nabla \cdot \boldsymbol{B})=0,
$$

is obtained. Thus, the numerical divergence of the magnetic field is not only advected outward with $\left|c_{\mathrm{h}}\right|$ unrelated to fluid flows but also diffused with the diffusivity $c_{\mathrm{p}}^{2}$.

\section{Numerical Experiments}

Typical two-dimensional MHD simulations of a compressible turbulence, the so-called Orszag-Tang vortex, are performed. The Poisson equation in the projection and face-projection methods is solved by the Jacobi method with a specific iteration number, typically 10,000, in all our tests because we reasonably compare the properties of both projection methods. The difference of the solution by each divergence-cleaning or divergence-free technique is not so clear except the hyperbolic divergence-cleaning method though the results are not shown here. In the hyperbolic divergence-cleaning method, fine structures are slightly spread out independent of $c_{\mathrm{p}}$. The numerical divergence of the magnetic field, on the other hand, is clearly different. The flux-CT and HLL-flux-CT methods limit the divergence errors to machine round-off. The divergence errors for the eight-wave method are larger than those for the other methods, where the maximum of the numerical divergence for the eight-wave method is more than ten times larger than that for the hyperbolic-divergence cleaning method. As shown in Fig. 2, the face-projection method seems to suppress the checkerboard instability, while the original projection method suffers from the odd-even decoupling in the divergence errors. We also find that the magnitudes of the divergence errors for the face-projection method are smaller than those for the projection method. The reason is believed to be that typical wave lengths of the Poisson equation for the face-projection method are shorter than those for the projection method, where, in general, short wavelength modes in the Poisson equation are converged faster than long wavelength modes.
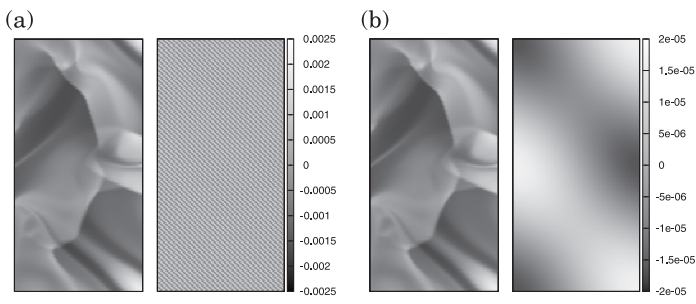

Fig. 2 Simulation results of the Orszag-Tang vortex. The left and right grayscale images show the two-dimensional distribution of the pressure and the discrete divergence of the magnetic field calculated by (a) the projection method and (b) the face-projection method, respectively. Grayscale bars are shown only for the discrete divergence, where the scales differ from one another.

We also perform an advection magnetic loop test as in Ref. [8]. In this test, a weak magnetic loop $\left(\beta=2 \times 10^{6}\right)$ is advected with fluid flows. Simulation results indicate that the magnetic loop is strongly damped by the hyperbolic divergence-cleaning method. On the other hand, the magnetic field is locally amplified and the shape of the magnetic loop is deformed by the flux-CT method. The other methods are presumed to be roughly acceptable compared with the upwind-CT method proposed by Ref. [8], although those methods also weakly deform the magnetic loop.

Finally, we perform extremely strong blast wave simulations, where $\boldsymbol{B}=(10 / \sqrt{2}, 10 / \sqrt{2}), \beta=2$ for $r \leq 0.125$ and $\beta=2 \times 10^{-3}$ for $r>0.125$. In general, it is very difficult to maintain the positivity of pressure at low $\beta$ shock fronts because large numerical errors and/or large numerical corrections of the magnetic field have a large effect on small thermal energy. Fig. 3 shows the pressure distribution calculated by each divergence-cleaning and divergence-free method. Notice that the grayscale in two-dimensional maps is fixed for a specific range, where high $\beta$ regions are not displayed to improve the visibility of low $\beta$ regions. We also notice that the scale of the vertical axis at the lower graphs differ from each other. Particularly, in this test, we impose an artificial lower limit on the pressure value because the present simulation settings may yield negative pressure. We find that the pressure reaches the lower limit in the hyperbolic divergencecleaning method, the flux-CT method, and the HLL-fluxCT method. The high pressure regions in those methods also seem to be modified unphysically. In particular, the pressure strongly increases along the shock front in the flux-CT method. Spurious oscillations are observed in the projection method because of the odd-even decoupling of the divergence errors, although the pressure does not become negative. Moreover, unphysical, low wave number mode structures seem to appear in the upwind side of the shock. On the other hand, spurious oscillations and negative pressures are not generated in either the faceprojection method or the eight-wave method. Because 


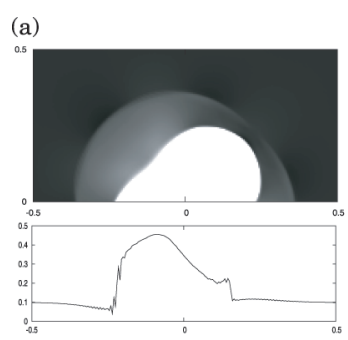

(b)
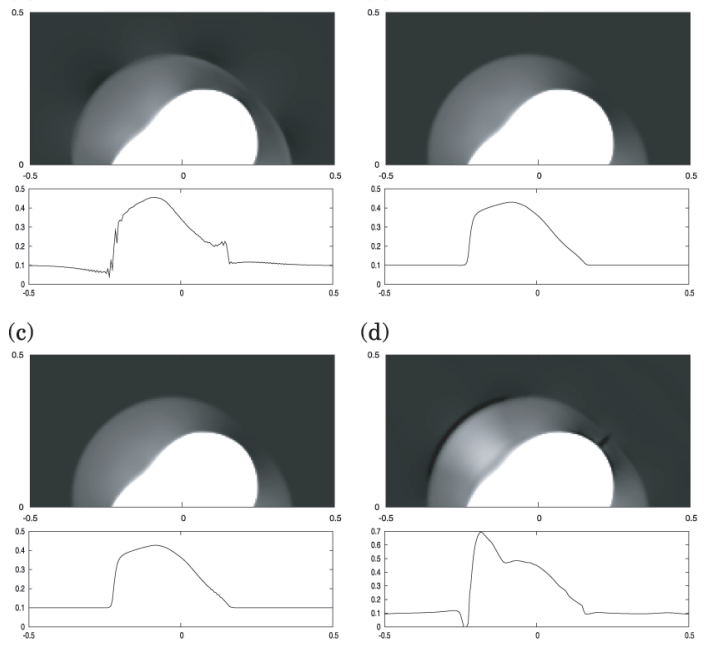

(d)

(e)
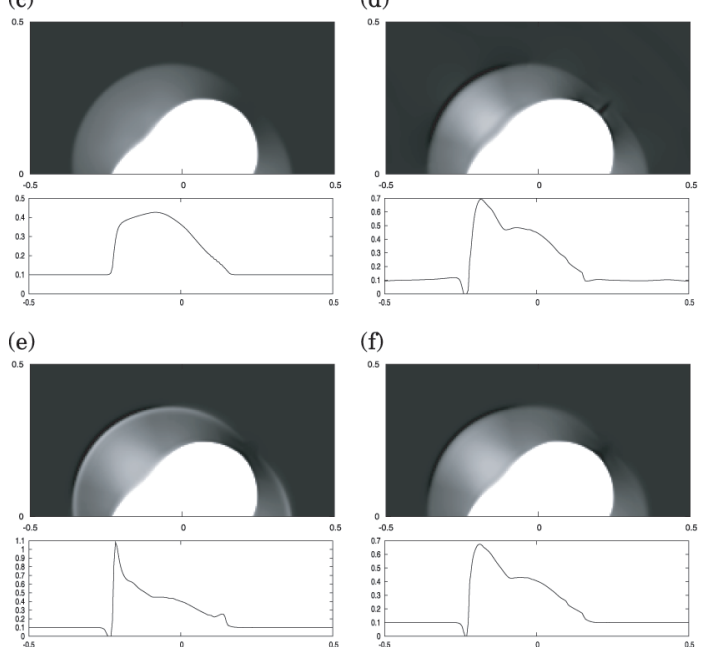

(f)

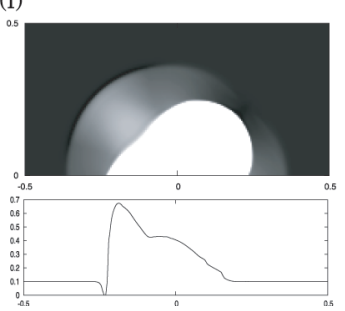

Fig. 3 Simulation results of the MHD blast wave. The upper grayscale images show the two-dimensional distribution of the pressure and the lower graphs indicate the pressure along $y=0.3$ calculated by (a) the projection method, (b) the face-projection method, (c) the eight-wave method, (d) the hyperbolic divergence-cleaning method, (e) the flux-CT method, and (f) the HLL-flux-CT method, respectively.

the projection method and the flux-CT and HLL-flux-CT methods vary the magnetic energy in the cell after the energy equation is solved, the pressure is directly affected by the reconstruction of the magnetic field at the cell center. Particularly in the low $\beta$ regions, the pressure may easily become negative. Therefore, we apply an energy correction here, as proposed by Ref. [11]:

$$
e^{\text {new }}=e^{\mathrm{G}}+\frac{1}{2}\left(\left|\boldsymbol{B}^{\mathrm{p}, \mathrm{c}}\right|^{2}-\left|\boldsymbol{B}^{\mathrm{G}}\right|^{2}\right),
$$

where $e^{\text {new }}, e^{\mathrm{G}}, \boldsymbol{B}^{\mathrm{p}}, \boldsymbol{B}^{\mathrm{c}}$, and $\boldsymbol{B}^{\mathrm{G}}$ are the corrected total energy, the total energy directly advanced by the Godunovtype schemes, the magnetic field at the cell center calculated by the projection method, the magnetic field at the cell center calculated by the flux-CT and HLL-flux-CT methods, and the magnetic field directly advanced by the Godunov-type schemes, respectively. We expect that the positivity property of the Godunov-type schemes will be maintained by the use of the energy correction even though the total energy is not conserved. Fig. 4 shows the pressure profile in a low $\beta$ region calculated using the projection method and the flux-CT and HLL-flux-CT methods with the energy correction. For comparison, the result obtained using the face-projection method without applying the en-

(a)

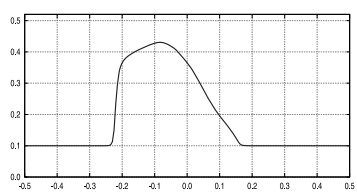

(c)

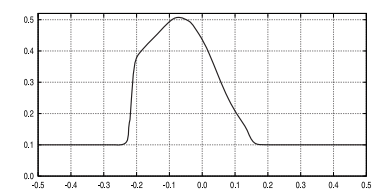

(b)

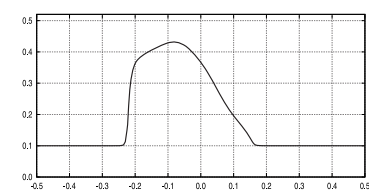

(d)

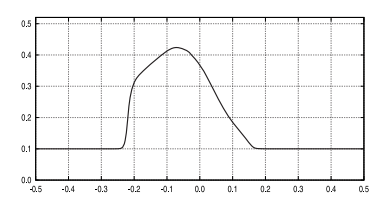

Fig. 4 Simulation results of the MHD blast wave. The graphs show the pressure along $y=0.3$ calculated by (a) the face-projection method, (b) the projection method with the energy correction, (c) the flux-CT method with the energy correction, and (d) the HLL-flux-CT method with the energy correction, respectively.

ergy correction is also shown. We find that the positivity of the pressure is recovered by the energy correction. The peak value and position of the pressure seem to be similar for each method with the exception of the peak value by the flux-CT method. It is thought that the flux-CT method may amplify some MHD waves as suggested by Ref. [8] and indicated by our numerical tests. On the other hand, the pressure jump at the shock front around $x \sim-0.2$ in the HLL-flux-CT method with the energy correction is slightly smaller than that in the other methods. Although the reason is not particularly obvious, wave speeds may slightly differ from each other. It is believed, however, that the difference is not significant.

\section{Conclusions}

Several important divergence-cleaning techniques as well as divergence-free techniques for multi-dimensional MHD schemes were comparatively investigated. In addition, we proposed a new technique, the so-called faceprojection method, that recovers the divergence-free field at the cell face using the projection technique before every time step. It was shown that the face-projection method may surpass the projection method in some respects. The HLL fluxes were also explicitly applied to the HLL-fluxCT method for the first time. Because the derivatives of the EMF at the cell face are estimated by an upwind-weighted average in this method, it may be weakly more diffusive than the upwind-CT method proposed by Ref. [8], in which the derivatives of the EMF at the cell face are replaced by those in the upwind side. However, the present method is expected to be more robust than the upwind-CT method. We also confirmed that the energy correction proposed by Ref. [11] works well for the projection, flux-CT, and HLLflux-CT methods for recovering the positivity in low $\beta$ regions. We believe that extensions of all present methods to three-dimensional and non-uniform grids are straightforward. 


\section{Acknowledgments}

This work was supported by a Grant-in-Aid for Young Scientists (B) No. 21740399 (Head Investigator: T. M.) from the Ministry of Education, Culture, Sports, Science and Technology (MEXT) of Japan and by a Grant-in-Aid for Scientific Research (B) No. 19340180 (Head Investigator: K. K.) from Japan Society for the Promotion of Science (JSPS).

[1] M. Brio and C.C. Wu, J. Comput. Phys. 75, 400 (1988).

[2] T. Miyoshi and K. Kusano, J. Comput. Phys. 208, 315 (2005).
[3] A. J. Ridley et al., Ann. Geophys. 28, 1589 (2010).

[4] D. Shiota et al., Astrophys. J. 718, 1305 (2010).

[5] G. Tóth, J. Comput. Phys. 161, 605 (2000).

[6] D.S. Balsara and J. Kim, Astrophys. J. 602, 1079 (2004).

[7] A. Dedner et al., J. Comput. Phys. 175, 645 (2002).

[8] T.A. Gardiner and J.M. Stone, J. Comput. Phys. 205, 509 (2005).

[9] C.-W. Shu and S. Osher, J. Comput. Phys. 77, 439 (1988).

[10] J.U. Brackbill and D.C. Barnes, J. Comput. Phys. 35, 426 (1980).

[11] D.S. Balsara and D.S. Spicer, J. Comput. Phys. 149, 270 (1999).

[12] K.G. Powell, ICASE Rep. 94-24, Langley, VA (1994). 